\title{
Value Distribution of L-Functions with Rational Moving Targets
}

\author{
Matthew Cardwell ${ }^{1}$, Zhuan Ye ${ }^{2 *}$ \\ ${ }^{1}$ Intelligent Medical Objects, Inc., Northbrook, USA \\ ${ }^{2}$ Department of Mathematical Sciences, Northern Illinois University, DeKalb, USA \\ Email: mcardwell@e-imo.com, *ye@math.niu.edu
}

Received August 26, 2013; revised September 26, 2013; accepted October 1, 2013

Copyright (C) 2013 Matthew Cardwell, Zhuan Ye. This is an open access article distributed under the Creative Commons Attribution License, which permits unrestricted use, distribution, and reproduction in any medium, provided the original work is properly cited. In accordance of the Creative Commons Attribution License all Copyrights (C) 2013 are reserved for SCIRP and the owner of the intellectual property Matthew Cardwell, Zhuan Ye. All Copyright (C) 2013 are guarded by law and by SCIRP as a guardian.

\begin{abstract}
We prove some value-distribution results for a class of $L$-functions with rational moving targets. The class contains Selberg class, as well as the Riemann-zeta function.
\end{abstract}

Keywords: Value Distribution; Moving Target; L-Function; Selberg Class

\section{Introduction}

We define the class $\mathcal{M}$ to be the collection of functions $L(s)=\sum_{n=1}^{\infty} a(n) / n^{s}$, satisfying Ramanujan hypothesis, Analytic continuation and Functional equation. We also denote the degree of a function $L \in \mathcal{M}$ by $d_{L}$ which is a non-negative real number. We refer the reader to Chapter six of [1] for a complete definitions. Obviously, the class $\mathcal{M}$ contains the Selberg class. Also every function in the class $\mathcal{M}$ is an $L$-function and the Riemann-zeta function is in the class. In this paper, we prove a value-distribution theorem for the class $\mathcal{M}$ with rational moving targets. The theorem generalizes the value-distribution results in Chapter seven of [1] from fixed targets to moving targets.

Theorem. Assume that $L \in \mathcal{M}$ and $R$ is a rational function with $\lim _{s \rightarrow \infty} R(s) \neq 1$. Let the roots of the equation $L(s)-R(s)=0$ be denoted by $\rho_{R}=\beta_{R}+i \gamma_{R}$. Then

$$
\begin{aligned}
& \text { (I) For any } b>\max \left\{\frac{1}{2}, 1-\frac{1}{d_{L}}\right\} \text {, } \\
& \qquad \sum_{\substack{\beta_{R}>b \\
T<\gamma_{R} \leq 2 T}}\left(\beta_{R}-b\right)=O(T), \text { as } T \rightarrow \infty .
\end{aligned}
$$

(II) For sufficiently large negative $b$,

$$
\begin{aligned}
& 2 \pi \sum_{T<\gamma_{R} \leq 2 T}\left(\beta_{R}-b\right)=(-b) d_{L} T \log \frac{4 T}{e}+O(\log T), \\
& \text { as } T \rightarrow \infty .
\end{aligned}
$$

Proof of (I). It is known that if $L \in \mathcal{M}$, then

$$
L(s)=\sum_{n=1}^{\infty} \frac{a(n)}{n^{s}}=1+O\left(k_{0}^{-\sigma}\right), \text { as } \sigma \rightarrow \infty ;
$$

where $k_{0}$ is the index of the first non-zero term of the sequence of $\{a(n)\}_{n=2}^{\infty}, s=\sigma+i$ with $\sigma, t \in \mathbb{R}$. Since $\lim _{\sigma \rightarrow \infty} L(s)-R(s) \neq 0$, there exists $\sigma_{0}>0$ such that $L(s)-R(s) \neq 0$ for $\operatorname{Re} s=\sigma>\sigma_{0}$. It follows that $\beta_{R}<\sigma_{0}$ for all real part of zeros of the function $L(s)-R(s)$. We set $R(z)=P(z) / Q(z)$ where the degrees of $P, Q$ are $p, q$, respectively; and define

$$
\tilde{\ell}(s)=(s)-R(s) \text {. }
$$

Thus, there is $r_{1}>1$ such that $\tilde{\ell}$ is analytic in the region $|s|>r_{1}$ since $L$ is a meromorphic function in $\mathbb{C}$ with the only pole at $s=1$. We apply Littlewood's argument principle [3] to $\tilde{\ell}$ in the rectangle $\mathcal{R}=\{\sigma+i t: b \leq \sigma \leq c, T \leq t \leq 2 T\} \quad$ where $\quad c, T$ are parameters satisfying $c>\max \left\{\sigma_{0}+1, b\right\}, T>r_{1}$. Thus,

$$
\int_{\partial \mathcal{R}} \log \tilde{\ell}(s) \mathrm{d} s=-2 \pi i \int_{b}^{c} v(\sigma, \mathcal{R}) \mathrm{d} \sigma
$$

where the given logarithm is defined as in Littlewood's argument principle [3]. To prove our result, however, we first decompose our auxiliary function by 


$$
\tilde{\ell}(s)=\left\{\begin{array}{l}
P(s)\left(\frac{L(s)}{P(s)}-\frac{1}{Q(s)}\right):=P(s) \ell_{1}(s) \text { for } p \leq q \\
R(s)\left(\frac{L(s)}{R(s)}-1\right):=R(s) \ell_{2}(s) \quad \text { for } p>q
\end{array}\right.
$$

Without loss of generality, we may assume that $p, q \geq 1$ whenever $p \leq q$ since we can always write
$R(s)=\left(P(s) s^{N}\right) /\left(Q(s) s^{N}\right)$ for $s \neq 0$ due to our choice of the parameters which define the rectangle $\mathcal{R}$. However, the modification will guarantee in the case of $k=1$ that $P, Q$ exhibit polynomial growth, which is necessary for our proof. In the case of $p>q, R$ already exhibits polynomial growth, and no such adjustment is necessary. We now integrate the logarithm of $\tilde{\ell}$ to get

$$
\int_{\partial \mathcal{R}} \log \tilde{\ell}(s) \mathrm{d} s= \begin{cases}\int_{\mathcal{O} \mathcal{R}} \log \ell_{1}(s)+\log P(s) \mathrm{d} s+O(T) & \text { for } p \leq q \\ \int_{\mathcal{L} \mathcal{R}} \log \ell_{2}(s)+\log R(s) \mathrm{d} s+O(T) & \text { for } p>q\end{cases}
$$

where the $O(T)$ terms are the integrals of the maximum contribution from writing $\tilde{\ell}(s)$ as a sum of logarithms. By our choice of $T$, both $\log P$ and $\log R$ are analytic in $\mathcal{R}$. Hence, Cauchy's Theorem gives

$$
\int_{\partial \mathcal{R}} \log \tilde{\ell}(s) \mathrm{d} s= \begin{cases}\int_{\partial \mathcal{R}} \log \ell_{1}(s) \mathrm{d} s+O(T) & \text { for } p \leq q \\ \int_{\partial \mathcal{R}} \log \ell_{2}(s) \mathrm{d} s+O(T) & \text { for } p>q\end{cases}
$$

To connect this integral with Littlewood's argument principle [3], we note that the definition of $c$ guaran- tees that

$$
\begin{aligned}
& -2 \pi i \int_{b}^{c} v(\sigma, \mathcal{R}) \mathrm{d} \sigma=-2 \pi i \sum_{\substack{\beta_{R}>b \\
T<\gamma_{R} \leq 2 T}} \int_{b}^{\beta_{R}} \mathrm{~d} \sigma \\
& =-2 \pi i \sum_{\substack{\beta_{R}>b \\
T<\gamma_{R} \leq 2 T}}\left(\beta_{R}-b\right) .
\end{aligned}
$$

In light of (2) and because the quantity given in (3) is imaginary-valued, we get for $k=1,2$

$$
\begin{aligned}
2 \pi i \sum_{\substack{\beta_{\beta_{R}>b} \\
T<\gamma_{k} \leq 2 T}}\left(\beta_{R}-b\right) \\
=i \operatorname{Im}\left[\int_{b}^{c} \log \left|\ell_{k}(\sigma+i T)\right|+i \arg \ell_{k}(\sigma+i T) \mathrm{d} \sigma+i \int_{T}^{2 T} \log \left|\ell_{k}(c+i t)\right|+i \arg \ell_{k}(c+i t) \mathrm{d} t\right. \\
\left.\quad-\int_{b}^{c} \log \left|\ell_{k}(\sigma+2 i T)\right|+i \arg \ell_{k}(\sigma+2 i T) \mathrm{d} \sigma-i \int_{T}^{2 T} \log \left|\ell_{k}(b+i t)\right|+i \arg \ell_{k}(b+i t) \mathrm{d} t\right]+O(T) \\
=-i\left[\int_{T}^{2 T} \log \left|\ell_{k}(b+i t)\right| \mathrm{d} t-\int_{T}^{2 T} \log \left|\ell_{k}(c+i t)\right| \mathrm{d} t-\int_{b}^{c} \arg \ell_{k}(\sigma+i T) \mathrm{d} \sigma+\int_{b}^{c} \arg \ell_{k}(\sigma+2 i T) \mathrm{d} \sigma\right]+O(T) \\
:=\sum_{j=1}^{4} I_{j, k}+O(T),
\end{aligned}
$$

for instance.

We now estimate $I_{1, k}$. For $T$ large enough, we have for $t \geq T, k=1$ (since $p, q \geq 1$ ),

$$
\begin{aligned}
\log \left|\ell_{1}(b+i t)\right| & =\log \left|\frac{L(b+i t)}{P(b+i t)}-\frac{1}{Q(b+i t)}\right| \leq \log \left(\left|\frac{L(b+i t)}{P(b+i t)}\right|+\frac{1}{\mid Q(b+i t)}\right) \\
& \leq \log (|L(b+i t)|+1)=\log ^{+}(|L(b+i t)|+1) \leq \log ^{+}|L(b+i t)|+\log 2 .
\end{aligned}
$$

Then for $T$ large enough, $t \geq T, k=2$, we find in a similar fashion that

$$
\begin{aligned}
\log \left|\ell_{2}(b+i t)\right|= & \log \left|\frac{L(b+i t)}{R(b+i t)}-1\right| \\
& \leq \log ^{+}|(b+i t)|+\log 2 .
\end{aligned}
$$

Since we have the same estimate for $k=1,2$, we find that

$$
\begin{aligned}
I_{1, k}(T, b) & =I_{1, k} \leq \int_{T}^{2 T} \log ^{+}|(b+i t)| \mathrm{d} t+O(T) \\
& =\frac{T}{2} \int_{T}^{2 T} \frac{\log ^{+}|(b+i t)|^{2}}{T} \mathrm{~d} t+O(T) \\
& \leq \frac{T}{2} \log ^{+}\left(\frac{1}{T} \int_{T}^{2 T}|L(b+i t)|^{2} \mathrm{~d} t\right)+O(T)
\end{aligned}
$$

where the final bound follows from Jensen's inequality. 
It is known [2] that for $b>\max \left\{\frac{1}{2}, 1-\frac{1}{d_{L}}\right\}$,

$$
\lim _{T \rightarrow \infty} \frac{1}{T} \int_{T}^{2 T}|L(b+i t)|^{2} \mathrm{~d} t=\sum_{n=1}^{\infty} \frac{|a(n)|^{2}}{n^{2 \sigma}}=O(1) .
$$

Hence, $I_{1, k}(T, b) \leq O(T)$ uniformly in

$b>\max \left\{\frac{1}{2}, 1-\frac{1}{d_{L}}\right\}$.

We next move to estimate $I_{2, k}$. For sufficiently large positive real number $c$, we have

$$
\left|\frac{L(c+i t)}{P(c+i t)}\right| \leq 1 \text { and }\left|\frac{L(c+i t)}{R(c+i t)}\right| \leq 1,
$$

so

$$
\log \left|\ell_{1}(c+i t)\right| \leq \log \left|1-\frac{L(c+i t)}{P(c+i t)}\right|
$$

since $q \geq 1$. Furthermore,

$$
\log \left|\ell_{2}(c+i t)\right|=\left|1-\frac{L(c+i t)}{R(c+i t)}\right| .
$$

Since we may take $c$ large enough so that $\left|\ell_{k}(c+i t)\right| \leq 1$, we may write $\log \ell_{k}(c+i t)$ using a Taylor series expansion in the rectangle $\mathcal{R}$. For $k=1$, we have after taking real parts that

$$
\begin{aligned}
& \log \left|\ell_{1}(c+i t)\right| \leq \operatorname{Re}\left(-\sum_{k=1}^{\infty} \frac{1}{k[P(c+i t)]^{k}}\left(\sum_{n=1}^{\infty} \frac{a(n)}{n^{c+i t}}\right)^{k}\right) \\
& =-\operatorname{Re}\left(\sum_{k=1}^{\infty} \frac{1}{k[P(c+i t)]^{k}} \sum_{n_{1}=1}^{\infty} \cdots \sum_{n_{k}=1}^{\infty} \frac{a\left(n_{1}\right) \cdots a\left(n_{k}\right)}{\left(n_{1} \cdots n_{k}\right)^{c+i t}}\right) .
\end{aligned}
$$

We now observe that for sufficiently large $T$ and some constant $M$ we have

$$
\int_{T}^{2 T}\left|\frac{t}{[P(c+i t)]^{k}\left(n_{1} \cdots n_{k}\right)^{i t}}\right| \leq \frac{T}{|P(c+i T)|^{k}} \leq M T^{1-k} \leq 1,
$$

for $k \in N$ and

$$
\limsup _{k \rightarrow \infty} \sqrt[k]{\frac{1}{k}\left(\sum_{n=1}^{\infty} \frac{1}{n^{c-\varepsilon}}\right)^{k}}=\sum_{n=1}^{\infty} \frac{1}{n^{c-\varepsilon}}<1
$$

for sufficiently large $c$. In light of these bounds and the definition of $\mathcal{M}$, we have (6)

where the last equality holds because $c$ could be sufficiently large. Replacing $P$ by $R$ in the above computations, we see analogously that $\left|I_{2,2}\right|=O(1)$.

Finally, we estimate $I_{3, k}$ and $I_{4, k}$. We show the computation for $I_{3, k}$ explicitly and note that the bound for $I_{4, k}$ follows analogously. We first suppose that $\ell_{k}(\sigma+i T)$ has exactly $N$ zeros for $b \leq \sigma \leq c$. Then, there are at most $N+1$ subintervals, counting for multiplicities, in which $\operatorname{Re}\left(\ell_{k}(\sigma+i T)\right)$ is of constant sign. Thus,

$$
\left|\arg \left(\ell_{k}(\sigma+i T)\right)\right| \leq(N+1) \pi .
$$

It remains to estimate $N$. To this end, we define

$$
g_{k}(z)=\frac{1}{2}\left(\ell_{k}(z+i T)+\overline{\ell_{k}(\bar{z}+i T)}\right) .
$$

Then

$$
g_{k}(\sigma)=\frac{1}{2}\left(\ell_{k}(\sigma+i T)+\overline{\ell_{k}(\sigma+i T)}\right)=\operatorname{Re} \ell_{k}(\sigma+i T),
$$

so that if $\ell_{k}(\sigma+i T)=0$ for $\sigma \in[b, c]$, then $g(\sigma)=0$.

Now let $R_{2}=c-b$ and $R^{\prime}>\max \left\{r_{1}, R_{2}\right\}$, and choose $T$ large enough so that $T>2 R^{\prime}$. Then $|z+i T|>R^{\prime}>R_{1}$ for $|z-c|<R^{\prime}$, showing that no zeros or poles of $\ell_{k}(z+i T)$ are located in $|z-c|<R^{\prime}$. Thus, both $\ell_{k}(z+i T)$ and $g_{k}(z)$ are analytic in $|z-c|<R^{\prime}$. Letting $\hat{n}_{c, k}(r)$ denote the number of zeros of $g_{k}(z)$ in $|z-c| \leq r$, we have

$$
\begin{aligned}
& \int_{0}^{2 R^{\prime}} \frac{\hat{n}_{c, k}(r)}{r} \mathrm{~d} r \geq \int_{R^{\prime}}^{2 R^{\prime}} \frac{\hat{n}_{c, k}(r)}{r} \mathrm{~d} r \\
& \geq \hat{n}_{c, k}\left(R^{\prime}\right) \int_{R^{\prime}}^{2 R^{\prime}} \frac{\mathrm{d} r}{r}=\hat{n}_{c, k}\left(R^{\prime}\right) \log 2 .
\end{aligned}
$$

By Jensen's formula

$\int_{0}^{2 R^{\prime}} \frac{\hat{n}_{c, k}(r)}{r} \mathrm{~d} r=\frac{1}{2 \pi} \int_{0}^{2 \pi} \log \left|g_{k}\left(c+2 R^{\prime} \mathrm{e}^{i \theta}\right)\right| \mathrm{d} \theta-\log \left|g_{k}(c)\right|$, and so

$$
\hat{n}_{c, k}\left(R^{\prime}\right) \leq \frac{1}{2 \pi \log 2} \int_{0}^{2 \pi} \log \left|g_{k}\left(c+2 R^{\prime} \mathrm{e}^{i \theta}\right)\right| \mathrm{d} \theta-\frac{\log \left|g_{k}(c)\right|}{\log 2} .
$$

$$
\begin{aligned}
\left|I_{2,1}\right| & =\left|-\operatorname{Re}\left(\sum_{k=1}^{\infty} \frac{1}{k} \sum_{n_{1}=1}^{\infty} \cdots \sum_{n_{k}=1}^{\infty} \frac{a\left(n_{1}\right) \cdots a\left(n_{k}\right)}{\left(n_{1} \cdots n_{k}\right)^{c}} \int_{T}^{2 T} \frac{\mathrm{d} t}{[P(c+i t)]^{k}\left(n_{1} \cdots n_{k}\right)^{i t}}\right)\right| \\
& \leq \sum_{k=1}^{\infty} \frac{1}{k} \sum_{n_{1}=1}^{\infty} \cdots \sum_{n_{k}=1}^{\infty}\left|\frac{a\left(n_{1}\right) \cdots a\left(n_{k}\right)}{\left(n_{1} \cdots n_{k}\right)^{c}}\right| \leq \sum_{k=1}^{\infty} \frac{1}{k}\left(\sum_{n=1}^{\infty} \frac{1}{n^{c-\varepsilon}}\right)^{k}=O(1),
\end{aligned}
$$


By (5), $\log \left|g_{k}(c)\right|$ is bounded. Further, it is clear from a property of $L$ functions that we have

$$
|L(s)| \leq A|t|^{B} \text {, as } t \rightarrow \infty \text {, as } t \rightarrow \infty \text {; }
$$

for some positive absolute numbers $A, B$ in any vertical strip of bounded width. The same estimate must hold for $g_{k}(z)$ as well. Thus, the integral in (8) is $O(\log T)$, implying that $\hat{n}_{c, k}(R)=O(\log T)$. Since the interval $[b, c] \subseteq D\left(c, R_{2}\right) \subseteq D\left(c, R^{\prime}\right)$, it follows that

$$
N \leq \hat{n}_{c, k}\left(R^{\prime}\right)=O(\log T) .
$$

With this bound, we integrate (7) to deduce that $\left|I_{3, k}\right| \leq \int_{b}^{c}\left|\arg \ell_{k}(\sigma+i t)\right| \mathrm{d} \sigma \leq \int_{b}^{c}(N+1) \pi \mathrm{d} \sigma=O(\log T)$.

As previously noted, we may bound $I_{4, k}$ in the same way. Thus, we attain the desired bounds for $j=1, \cdots, 4$ and $k=1,2$. Consequently, the first part of the theorem is proved by using (4).

Proof of (II). As in the proof of the first part of the theorem, we conclude that there exists a real number $\sigma_{0}$ for which the real parts $\beta_{R}$ of all $R$-values satisfy $\beta_{R}<\sigma_{0}$; and also, there exist $B, T^{\prime}>0$ for each rational function $R$ such that no zeros of

$L(s)-R(s)=0$ lie in the quarter-plane $\sigma<-B, t>T^{\prime}$. As before, we define the rectangle

$\mathcal{R}=\{s=\sigma+i t: b \leq \sigma \leq c, T \leq t \leq 2 T\}$ where $b, c, T$ are parameters satisfying $b<-B-1, c>\max \left\{\sigma_{0}+1, b\right\}, T>\max \left\{r_{1}, T^{\prime}+1\right\}$.

Proceeding as in the proof of the first part of the theorem, we see that

$$
\begin{aligned}
2 \pi i \sum_{T<\gamma_{R} \leq 2 T}\left(\beta_{R}-b\right) & =-i\left[\int_{T}^{2 T} \log |\tilde{\ell}(b+i t)| \mathrm{d} t-\int_{T}^{2 T} \log \left|\ell_{k}(c+i t)\right| \mathrm{d} t\right. \\
& \left.=\int_{b}^{c} \arg \ell_{k}(\sigma+i T) \mathrm{d} \sigma+\int_{b}^{c} \arg \ell_{k}(\sigma+2 i T) \mathrm{d} \sigma\right]+O(T) \\
& :=I_{1}+\sum_{j=2}^{4} I_{j, k}+O(T)
\end{aligned}
$$

for $k=1,2$ where $\ell_{k}$ is defined as in (1). In the equation above, we note that we have chosen to compute $I_{1}$ separately. Indeed, this is the only estimate that we will need. For the integrals $I_{j, k}, j=2,3,4$ and $k=1,2$, the bounds given as in the proof of the first part of the theorem still hold. First, integral $I_{2, k}$ is unchanged. On the other hand, the integrals $I_{3, k}, I_{4, k}$ have changed by our choice of $b$, but, as we have done as before, we still have the desired bound since the only requirement is that we consider $L$ in a vertical strip of fixed width, which we have in this case.

We now bound $I_{1}$. Since $b<-B$, we have by the functional equation in the definition of $L$ function,

$$
\begin{aligned}
|L(s)-R(s)| & =\left|\Lambda_{L}(s) \overline{L(1-\bar{s})}-R(s)\right|=\left|\Lambda_{L}(s)\right||L(1-\bar{s})|\left|1-\frac{R(s)}{\Lambda_{L}(s) L(1-\bar{s})}\right| \\
& =\left|\Lambda_{L}(s)\right||L(1-\bar{s})|\left|1-\frac{R(s)}{L(s)}\right| .
\end{aligned}
$$

Taking logarithms, we get

$$
\begin{aligned}
& \log |L(s)-R(s)| \\
& =\log \left|\Lambda_{L}(s)\right|+\log |\overline{L(1-\bar{s})}|+\log \left|1-\frac{R(s)}{L(s)}\right| .
\end{aligned}
$$

Since, for $t>1$, we have, uniformly in $\sigma$,

$$
\begin{aligned}
& \log \left|\Lambda_{L}(s)\right| \\
& =\left(\lambda Q^{2} t^{d_{L}}\right)^{\frac{1}{2}-\sigma-i t} \exp \left(i t d_{L}+\frac{i \pi\left(\mu-d_{L}\right)}{4}\right)\left(1+O\left(\frac{1}{t}\right)\right),
\end{aligned}
$$

where $\mu, \lambda$ are two constants. It follows, for $s=\sigma+i t$ as $t \rightarrow \infty$, that

$$
\begin{aligned}
\log \left|\Lambda_{L}(s)\right|= & \log \left|\left(\lambda Q^{2} t^{d_{L}}\right)^{\frac{1}{2}-\sigma-i t} \exp \left(i t+\frac{i \pi\left(\mu-d_{L}\right)}{4}\right)\left(1+O\left(\frac{1}{t}\right)\right)\right| \leq\left(\frac{1}{2}-\sigma\right) \log \left|\lambda Q^{2} t^{d_{L}}\right| \\
& -\log \left|\left(\lambda Q^{2} t^{d_{L}}\right)^{i t}\right|+\log \left|1+O\left(\frac{1}{t}\right)\right|=\left(\frac{1}{2}-\sigma\right)\left(d_{L} \log t+\log \left(\lambda Q^{2}\right)\right)+O\left(\frac{1}{t}\right) .
\end{aligned}
$$


We now consider the last term in (9). Since,

$$
\limsup _{t \rightarrow \pm \infty} \frac{\log |L(b+i t)|}{\log |t|}=\left(\frac{1}{2}-b\right) d_{L},
$$

and noting $b<0$, we have for any $\delta>0$ and $t \geq T$

$$
|L(b+i t)| \geq|t|^{\left(\frac{1}{2}-b\right) d_{L}-\delta}
$$

for sufficiently large $T$. Then we see the quotient

$$
\left|\frac{R(b+i t)}{L(b+i t)}\right| \leq\left|\frac{R(b+i t)}{\left(\frac{1}{2}-b\right) d_{L}-\delta}\right|=O\left(\frac{1}{t}\right)
$$

when $-b$ is large enough so that

$$
\operatorname{deg} R<\left(\frac{1}{2}-b\right) d_{L}-\delta+1
$$

Therefore, we find that

$$
\log \left|1-\frac{R(s)}{L(s)}\right|=O\left(\frac{1}{t}\right) .
$$

Integrating in light of these estimates, we see

$$
\begin{aligned}
& \int_{T}^{2 T} \log |L(b+i t)-R(b+i t)| \mathrm{d} t \\
& =\left(\frac{1}{2}-b\right) \int_{T}^{2 T}\left(d_{L} \log t+\log \left(\lambda Q^{2}\right)\right) \mathrm{d} t \\
& \quad+\int_{T}^{2 T} \log |L(1-b-i t)| \mathrm{d} t+O(\log T) .
\end{aligned}
$$

The first integral is $d_{L} T \log \frac{4 T}{e}+T \log \left(\lambda Q^{2}\right)$, and the second integral is $O(1)$ for sufficiently large and negative $b$ by the method used to derive (6). Hence,

$$
I_{1}=\left(\frac{1}{2}-b\right)\left(d_{L} T \log \frac{4 T}{e}+T \log \left(\lambda Q^{2}\right)\right)+O(\log T) .
$$

With the estimates for the $I_{j, k}$ 's, we have proved the second part of the theorem.

\section{REFERENCES}

[1] J. Steuding, "Value Distribution of L-Functions," Number 1877 in Lecture Notes in Mathematics, Springer, 2007.

[2] H. S. A. Potter, "The Mean Values of Certain Dirichlet Series I," Proceedings London Mathematical Society, Vol. 46, No. 2, 1940, pp. 467-468. http://dx.doi.org/10.1112/plms/s2-46.1.467

[3] E. C. Titchmarsh, "The Theory of Functions," 2nd Edition, Oxford, 1939. 